\title{
Estimation of the Daily Recovery Cases in Egypt for COVID-19 Using Power Odd Generalized Exponential Lomax Distribution
}

\author{
Hanem Mohamed ${ }^{1}$ (D) Salwa A. Mousa ${ }^{2}$ - Amina E. Abo-Hussien ${ }^{2}$. \\ Magda M. Ismail ${ }^{1}$
}

Received: 2 October 2020 / Revised: 23 March 2021 / Accepted: 15 April 2021 /

Published online: 6 September 2021

(c) The Author(s), under exclusive licence to Springer-Verlag GmbH Germany, part of Springer Nature 2021

\begin{abstract}
Covid-19 has become an important topic this days, because of its bad effect in many fields such as Economics, industrial and commerce. In this paper, Covid-19 will be studied statistically point of view depending on the recovery cases in the Arab Republic of Egypt in the interval of (20 March to 20 August 2020). A power odd generalized exponential Lomax distribution has been considered. Some mathematical properties of the distribution are studied. The method of maximum likelihood and maximum product of spacings are used for estimating the model parameters. Also 95\% asymptotic confidence intervals for the estimates of the parameters are derived. A simulation study was conducted to evaluate the numerical behavior of the estimates. The proposed methods are utilized to find estimates of the parameters of power odd generalized exponential Lomax distribution for the recovery cases of corona virus in Egypt.
\end{abstract}

Keywords COVID-19 - Power odd generalized exponential Lomax distribution (POGEL) · Maximum likelihood estimation (ML) · Maximum product spacings (MPS)

\section{Introduction}

Coronavirus disease (COVID-19) is an infectious disease caused by a newly discovered coronavirus. Most people infected with the COVID-19 virus will experience mild to moderate respiratory illness and recover without requiring special treatment.

Hanem Mohamed

nonaeslam97@gmail.com

1 Faculty of Commerce, Al-Azhar University, Cairo, Egypt

2 Faculty of Commerce, (Girls Campus), Al-Azhar University, Cairo, Egypt 
Older people and those with underlying medical problems like cardiovascular disease, diabetes, chronic respiratory disease, and cancer are more likely to develop serious illness.

Data science is a concept to unify statistics, data analysis, informatics, and their related methods in order to understand and analyze actual phenomena with data.. Several researchers concerned with finding many ways to study different types of data $[1,2]$. This paper is introducing a statistical technique to study the COVID-19 $[3,4]$ which appeared at the end of 2019 in China, and in a few weeks, it spread in other countries worldwide, including Europe, USA and Africa [5, 6]. Some statisticians have been studying COVID-19 in some ways, among of them studying the COVID-19 time series to understand the growth behavior of COVID-19 cases series [7], Using generalized logistics regression to forecast population infected by Covid-19 [8]. In this paper, the reported COVID-19 number of recovery cases in Egypt, for the period 20March 2020 to 20 August 2020, is modeled using statistical distributions.

In recent years there are several new families of probability distributions which are proposed by several authors. Such families have great flexibility and generalize many well-known distributions. So several classes have been proposed, in the statistical literature, by adding one or more parameters to generate new distributions. Among this literature exponential Lomax [9], exponentiated Weibull- Lomax [10], the odd Lomax generator [11], the generalized odd inverted exponential-G family [12], the odd log-logistic Lindley-G [13] and the odd Dagum family of distributions [14].

The generalized exponential and Lomax distributions are two important distributions in studies and practice. These distributions have several important statistical properties [15]. Introduced the generalized exponential distribution and derived some properties of this distribution [16]. The Lomax distribution is introduced as an important model for lifetime analysis, it is also called Pareto type II distribution. The distribution is widely used in several fields such as business and econometrics [17]. [18] Proposed a new generalized family of distributions called T-X family. The T-X family consists of many sub-families of distributions. Based on this technique, one can develop new distributions that may be very general and flexible or for fitting specific types of data distributions such as highly left-tailed, right-tailed, thin-tailed, or heavy-tailed distributions as well as bimodal distributions.

Using the generalized exponential and Lomax distribution, the odd generalized exponential Lomax distribution (OGEL) based on the $T-X$ family can be obtained [19].

A random variable $\mathrm{X}$ has the odd generalized exponential Lomax distribution with four parameter $\lambda, \gamma, \beta$ and $\theta$ if it has the pdf and cdf given by:

$$
\begin{aligned}
\mathrm{f}(x ; \lambda, \gamma, \beta, \theta)= & \frac{\lambda \gamma \beta}{\theta}\left(1+\frac{x}{\theta}\right)^{\beta-1} e^{-\lambda\left[\left(1+\frac{x}{\theta}\right)^{\beta}-1\right]} \\
& {\left[1-e^{-\lambda\left[\left(1+\frac{x}{\theta}\right)^{\beta}-1\right]}\right]^{\gamma-1}, x>0, \lambda, \gamma, \beta>0, \theta>1 . }
\end{aligned}
$$




$$
F(\mathrm{x} ; \lambda, \gamma, \beta, \theta)=\left[1-\mathrm{e}^{-\lambda\left[\left(1+\frac{\mathrm{x}}{\theta}\right)^{\beta}-1\right]}\right]^{\gamma}
$$

The rest of the paper is unfolded as follows: organized as follows: In Sect. 2 The density function of the POGEL distribution is derived. The main descriptive properties are introduced in Sect. 3. In Sect. 4 some of the special cases are obtained. The ML and the MPS of the parameters are discussed in Sect. 5. A simulation study is tabulated and discussed in Sect. 6. A real life application is presented in Sect. 7 while some concluding remarks are given in Sect. 8.

\section{The Power Odd Generalized Exponential Lomax Distribution}

[20] introduced an extension of Lindley distribution by using this transformation $\mathrm{x}=\mathrm{t}^{\delta}$ hence; it is of interest to know what would be the distribution of similar power transformation of odd generalized exponential Lomax distribution by using the transformation.

Based on the transformation $\mathrm{x}=\mathrm{t}^{\delta}$ family, the proposed distribution is derived by replacing $\mathrm{x}=\mathrm{t}^{\delta}$ in (1) as follows

$$
\begin{aligned}
f(t ; \lambda, \gamma, \beta, \delta, \theta)= & \frac{\gamma \beta \delta \lambda}{\theta} t^{\delta-1}\left(1+\frac{t^{\delta}}{\theta}\right)^{\beta-1} e^{-\lambda\left[\left(1+\frac{t^{\delta}}{\theta}\right)^{\beta}-1\right]} \\
& {\left[1-e^{-\lambda\left[\left(1+\frac{t^{\delta}}{\theta}\right)^{\beta}-1\right]}\right]^{\gamma-1}, t>0, \lambda, \beta, \gamma, \delta>0, \theta>1 . }
\end{aligned}
$$

The resulting distribution will be referred to as the power odd generalized exponential Lomax distribution (POGEL).

Then the cdf of the distribution is as follows:

$$
F(t ; \lambda, \gamma, \beta, \delta, \theta)=\left[1-e^{-\lambda\left[\left(1+\frac{t^{\delta}}{\theta}\right)^{\beta}-1\right]}\right]^{\gamma}, t>0, \lambda, \beta, \gamma, \delta>0, \theta>1
$$

\section{Some Descriptive Properties of the POGEL Distribution}

This Section provides some properties of the POGEL distribution.

I. Main properties of the POGEL

a. The survival function denoted by $S(t)$, is given by: 


$$
S(t)=1-F(t)=1-\left[1-\mathrm{e}^{-\lambda\left[\left(1+\frac{t^{\delta}}{\theta}\right)^{\beta}-1\right]}\right]^{\gamma} .
$$

b. The hazard rate function, $\mathrm{h}(\mathrm{t})$, is given by:

$$
h(t)=\frac{\lambda \gamma \beta \delta t^{\delta-1}\left(1+\frac{t^{\delta}}{\theta}\right)^{\beta-1} e^{-\lambda\left[\left(1+\frac{t^{\delta}}{\theta}\right)^{\beta}-1\right]}\left[1-e^{-\lambda\left[\left(1+\frac{t^{\delta}}{\theta}\right)^{\beta}-1\right]}\right]^{\gamma-1}}{\left.\theta\left[1-\left[1-e^{-\lambda\left[\left(1+\frac{t^{\delta}}{\theta}\right)^{\beta}-1\right.}\right]\right]^{\gamma}\right]}
$$

c. The reversed hazard rate function, $r(t)$, is given by:

$$
r(t)=\frac{\frac{\gamma \beta \delta \lambda}{\theta} t^{\delta-1}\left(1+\frac{t^{\delta}}{\theta}\right)^{\beta-1} e^{-\lambda\left[\left(1+\frac{t^{\delta}}{\theta}\right)^{\beta}-1\right]}}{1-e^{-\lambda\left[\left(1+\frac{t^{\delta}}{\theta}\right)^{\beta}-1\right]}}
$$

d. The cumulative hazard rate function, $\mathrm{H}(\mathrm{t})$, is given by:

$$
H(t)=-\ln \left[1-\left[1-\mathrm{e}^{-\lambda\left[\left(1+\frac{t^{\delta}}{\theta}\right)^{\beta}-1\right]}\right]^{\gamma}\right]
$$

e. Quantiles and median of the POGEL distribution

The quantile function $\left(t_{q}\right)$, is given by:

$$
t_{q}=\left[\theta\left\{\left[1-\frac{1}{\lambda} \ln \left[1-\left(1-q^{\frac{1}{\gamma}-1}\right)\right]^{\frac{1}{\beta}}\right]-1\right\}\right]^{\frac{1}{\delta}}
$$

In particular when $q=0.5$ the median of the POGEL distribution is given by:

$$
t_{0.5}=\left[\theta\left\{\left[1-\frac{1}{\lambda} \ln \left[1-\left(1-(0.5)^{\frac{1}{\gamma}-1}\right)\right]^{\frac{1}{\beta}}\right]-1\right\}\right]^{\frac{1}{\delta}}
$$

And the Inter-Quantile Range (IQR) which is defined as the difference between the third quartile and the first quartile can be expressed as: 


$$
\begin{aligned}
\operatorname{IQR}(t)= & {\left[\boldsymbol{\theta}\left\{\left[1-\frac{1}{\lambda} \ln \left[1-\left(1-(0.75)^{\frac{1}{\gamma}-1}\right)\right]^{\frac{1}{\beta}}\right]-1\right\}\right]^{\frac{1}{\delta}} } \\
& -\left[\theta\left\{\left[1-\frac{1}{\lambda} \ln \left[1-\left(1-(0.25)^{\frac{1}{\gamma}-1}\right)\right]^{\frac{1}{\beta}}\right]-1\right\}\right]^{\frac{1}{\delta}}
\end{aligned}
$$

f. Some useful expansion for POGEL distribution

An expansion for pdf is derived.

$$
\begin{aligned}
& \text { Since } 0<\left[1-e^{-\lambda\left[\left(1+\frac{t^{\delta}}{\theta}\right)^{\beta}-1\right]}\right]\langle 1 \text { for } t\rangle 0 \text {, we obtain } \\
& {\left[1-e^{-\lambda\left[\left(1+\frac{t^{\delta}}{\theta}\right)^{\beta}-1\right]}\right]^{\gamma-1}=\sum_{i=0}^{\gamma-1}\left(\begin{array}{c}
\gamma-1 \\
i
\end{array}\right)(-1)^{i} e^{-\lambda i\left[\left(1+\frac{t^{\delta}}{\theta}\right)^{\beta}-1\right]}} \\
& \text { Using series expansion of }\left\{e^{-\lambda(i+1)\left[\left(1+\frac{t^{\delta}}{\theta}\right)^{\beta}-1\right]}\right\} \text { and substituting from }
\end{aligned}
$$
into (3), to get

$$
\begin{aligned}
f(t: \lambda, \gamma, \beta, \delta, \theta)= & \sum_{j=0}^{\infty} \sum_{i=0}^{\gamma-1}\left(\begin{array}{c}
\gamma-1 \\
i
\end{array}\right)(-1)^{i+j} \frac{\lambda^{j} \gamma \beta \delta(i+1)^{j}}{\theta(j) !} t^{\delta-1}\left(1+\frac{x}{\theta}\right)^{\beta-1} \\
& {\left[\left(1+\frac{t^{\delta}}{\theta}\right)^{\beta}-1\right]^{j}\left(1+\frac{t^{\delta}}{\theta}\right)^{\beta-1} . }
\end{aligned}
$$

Using binomial expansion of $\left[\left(1+\frac{t^{\delta}}{\theta}\right)^{\beta}-1\right]^{j}$ we obtain

$\left[\left(1+\frac{t^{\delta}}{\theta}\right)^{\beta}-1\right]^{j}=\sum_{k=0}^{j}\left(\begin{array}{l}j \\ k\end{array}\right)(-1)^{k}\left[\left(1+\frac{t^{\delta}}{\theta}\right)^{\beta}\right]^{j-k}$

Substituting from (14) into (13), we get

$$
\begin{aligned}
f(t: \lambda, \gamma, \beta, \delta, \theta)= & \sum_{j=0}^{\infty} \sum_{i=0}^{\gamma-1} \sum_{k=0}^{j}\left(\begin{array}{c}
\gamma-1 \\
i
\end{array}\right)\left(\begin{array}{l}
j \\
k
\end{array}\right)(-1)^{i+j+k} \frac{\lambda^{j} \gamma \beta \delta(i+1)^{j}}{\theta(j) !} t^{\delta-1} \\
& \left(1+\frac{t^{\delta}}{\theta}\right)^{\beta(j-k+1)-1}, t>0, \lambda, \beta, \gamma, \delta>0, \theta>1 .
\end{aligned}
$$

g. The $r^{\text {th }}$ moment is given by:

The $r^{\text {th }}$ non-central moment of POGEL distribution is given by

$\underset{r}{\mu}=\sum_{j=0}^{\infty} \sum_{i=0}^{\gamma-1} \sum_{k=0}^{j}\left(\begin{array}{c}\gamma-1 \\ i\end{array}\right)\left(\begin{array}{c}j \\ k\end{array}\right)(-1)^{i+j+k} \frac{\lambda^{j} \gamma \beta \delta(i+1)^{j}}{\theta(j) !} \int_{0}^{\infty} t^{r} t^{\delta-1}\left(1+\frac{t^{\delta}}{\theta}\right)^{\beta(j-k+1)-1} d t$, 
Then, the $r^{\text {th }}$ moment of POGEL under the condition $(\beta j+r+1)<\beta(k+1)$ is derived as follows:

$$
\mu_{r}{ }^{3}=\sum_{j=0}^{\infty} \sum_{i=0}^{\gamma-1} \sum_{k=0}^{j}\left(\begin{array}{c}
\gamma-1 \\
i
\end{array}\right)\left(\begin{array}{l}
j \\
k
\end{array}\right)(-1)^{i+j+k} \frac{\lambda^{j} \gamma \beta \delta(i+1)^{j} \theta^{\frac{r}{\delta}}}{(j) !} \beta\left[\left(\frac{r}{\delta}+1\right), \beta(j-k+1)-(r+1)\right] .
$$

Depending on Eq. (16), the basic statistical properties of POGEL are.

The mean and the variance, of the POGEL distribution are, respectively, given by:

$$
E(t)=\sum_{j=0}^{\infty} \sum_{i=0}^{\gamma-1} \sum_{k=0}^{j}\left(\begin{array}{c}
\gamma-1 \\
i
\end{array}\right)\left(\begin{array}{l}
j \\
k
\end{array}\right)(-1)^{i+j+k} \frac{\lambda^{j} \gamma \beta \delta(i+1)^{j} \theta^{\frac{1}{\delta}}}{(j) !} \beta\left[\left(\frac{1}{\delta}+1\right), \beta(j-k+1)-(2)\right]
$$

And

$$
\begin{aligned}
\operatorname{Var}(t)= & \sum_{j=0}^{\infty} \sum_{i=0}^{\gamma-1} \sum_{k=0}^{j}\left(\begin{array}{c}
\gamma-1 \\
i
\end{array}\right)\left(\begin{array}{l}
j \\
k
\end{array}\right)(-1)^{i+j+k} \frac{\lambda^{j} \gamma \beta \delta(i+1)^{j} \theta^{\frac{2}{\delta}}}{(j) !} \beta\left[\left(\frac{2}{\delta}+1\right), \beta(j-k+1)-(3)\right] \\
& -\left[\sum_{j=0}^{\infty} \sum_{i=0}^{\gamma-1} \sum_{k=0}^{j}\left(\begin{array}{c}
\gamma-1 \\
i
\end{array}\right)\left(\begin{array}{c}
j \\
k
\end{array}\right)(-1)^{i+j+k} \frac{\lambda^{j} \gamma \beta \delta(i+1)^{j} \theta^{\frac{1}{\delta}}}{(j) !} \beta\left[\left(\frac{1}{\delta}+1\right), \beta(j-k+1)-(2)\right]\right]^{2} .
\end{aligned}
$$

h. The moment generating function is given by:

$$
\begin{aligned}
M_{x}(t)= & \sum_{j=0}^{\infty} \sum_{r=0}^{\infty} \sum_{i=0}^{\gamma-1} \sum_{k=0}^{j}\left(\begin{array}{c}
\gamma-1 \\
i
\end{array}\right)\left(\begin{array}{l}
j \\
k
\end{array}\right)(-1)^{i+j+k} \frac{\lambda^{j} \gamma \beta(i+1)^{j} \theta^{\frac{r}{\delta}} t^{r}}{(j) !(r) !} \\
& \beta\left[\frac{r}{\delta}+1, \beta(j-k+1)-(r+1)\right] .
\end{aligned}
$$

i. Order statistics

Let $T_{(1: n)}, T_{(2: n)}, \ldots, T_{(n: n)}$ denote the order statistics obtained for a random sample $t_{1}, t_{2}, \ldots, t_{n}$ from POGEL distribution with cdf (4) and pdf (3). The pdf of $r^{\text {th }}$ order statistics is defined by:

$\mathrm{f}_{\mathrm{r}, \mathrm{n}}(\mathrm{t} ; \omega)=\frac{1}{\mathrm{~B}(\mathrm{r}, \mathrm{n}-\mathrm{r}+1)} \mathrm{f}(\mathrm{t} ; \omega)[\mathrm{F}(\mathrm{t} ; \omega)]^{\mathrm{r}-1}[1-\mathrm{F}(\mathrm{t} ; \omega)]^{\mathrm{n}-\mathrm{r}}$

Using binomial expansion

$$
f_{r, n}(t ; \omega)=\frac{1}{B(r, n-r+1)} \sum_{j=0}^{n-r}\left(\begin{array}{c}
n-r \\
j
\end{array}\right)(-1)^{j} f(t ; \omega)[F(t ; \omega)]^{j+r-1}
$$

Substituting (3) and (4) in (20), as follows: 


$$
\begin{gathered}
\mathrm{f}_{\mathrm{r}, \mathrm{n}}(\mathrm{t} ; \omega)=\frac{1}{\mathrm{~B}(\mathrm{r}, \mathrm{n}-\mathrm{r}+1)} \sum_{\mathrm{j}=0}^{\mathrm{n}-\mathrm{r}}\left(\begin{array}{c}
\mathrm{n}-\mathrm{r} \\
\mathrm{j}
\end{array}\right)(-1)^{\mathrm{j}} \frac{\gamma \beta \delta \lambda}{\theta} \mathrm{t}^{\delta-1}\left(1+\frac{\mathrm{t}^{\delta}}{\theta}\right)^{\beta-1} \\
\mathrm{e}^{-\lambda\left[\left(1+\frac{\mathrm{t}^{\delta}}{\theta}\right)^{\beta}-1\right]}\left[1-\mathrm{e}^{\left.-\lambda\left[\left(1+\frac{\delta^{\delta}}{\theta}\right)^{\beta}-1\right]\right]^{\gamma(\mathrm{r}+\mathrm{j}-1)}}\right. \\
\text { Since } 1-e^{-\lambda\left[\left(1+\frac{t^{\delta}}{\theta}\right)^{\beta}-1\right]}=\sum_{i=0}^{\gamma(r+j-1}\left(\begin{array}{c}
\gamma(r+j-1) \\
i
\end{array}\right)(-1)^{i} e^{-\lambda i\left[\left(1+\frac{t^{\delta}}{\theta}\right)^{\beta}-1\right]} .
\end{gathered}
$$

Then

$$
\begin{aligned}
f_{r, n}(t ; \omega)= & \frac{1}{B(r, n-r+1)} \sum_{i=0}^{n-r} \sum_{i=0}^{\gamma(r+j-1)}\left(\begin{array}{c}
\gamma(r+j-1) \\
i
\end{array}\right)\left(\begin{array}{c}
n-r \\
j
\end{array}\right)(-1)^{i+j} \\
& \frac{\gamma \beta \delta \lambda}{\theta} t^{\delta-1}\left(1+\frac{t^{\delta}}{\theta}\right)^{\beta-1} e^{-\lambda(i+1)\left[\left(1+\frac{t^{\delta}}{\theta}\right)^{\beta}-1\right]}
\end{aligned}
$$

In particular, the pdf of the smallest order statistics is obtained by substituting $\mathrm{r}=1$ in (22) as follows:

$$
\left.\mathrm{f}_{1, \mathrm{n}}(\mathrm{t} ; \omega)=\mathrm{n} \sum_{\mathrm{i}=0}^{\mathrm{n}-1} \sum_{\mathrm{i}=0}^{\gamma \mathrm{j}}\left(\begin{array}{c}
\gamma \mathrm{j} \\
\mathrm{i}
\end{array}\right)\left(\begin{array}{c}
\mathrm{n}-1 \\
\mathrm{j}
\end{array}\right)(-1)^{\mathrm{i}+\mathrm{j}} \frac{\gamma \beta \delta \lambda}{\theta} \mathrm{t}^{\delta-1}\left(1+\frac{\mathrm{t}^{\delta}}{\theta}\right)^{\beta-1} \mathrm{e}^{-\lambda(\mathrm{i}+1)\left[\left(1+\frac{\mathrm{t}^{\delta}}{\theta}\right)^{\beta}-1\right.}\right] \text {. }
$$

Also, the pdf of largest order statistics is obtained by substituting $r=n$ in (22) as follows:

$$
f_{n, n}(t ; \omega)=n \sum_{i=0}^{\gamma(n+j-1)}\left(\begin{array}{c}
\gamma(n+j-1) \\
i
\end{array}\right)(-1)^{i+j} \frac{\gamma \beta \delta \lambda}{\theta} t^{\delta-1}\left(1+\frac{t^{\delta}}{\theta}\right)^{\beta-1} e^{-\lambda(i+1)\left[\left(1+\frac{t^{\delta}}{\theta}\right)^{\beta}-1\right]} .
$$

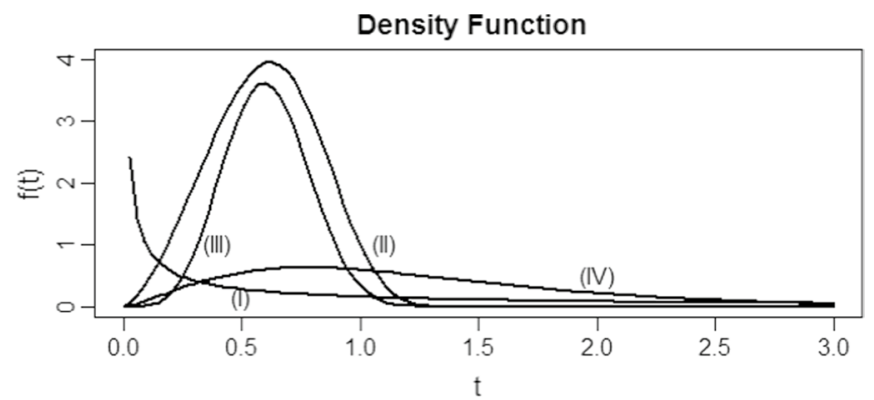

Fig (1.I): $(\lambda=3, \gamma=3, \beta=3, \delta=2, \theta=2)$,

Fig (1.II): $(\lambda=2, \gamma=2.5, \beta=1.5, \delta=1, \theta=2.5)$, Fig (1.III): $(\lambda=0.3, \gamma=0.25, \beta=1.2, \delta=1, \theta=1.5), \quad$ Fig (1.IV): $(\lambda=1.5, \gamma=1.5, \beta=2, \delta=3, \theta=1.5)$.

Fig. 1 pdf of POGEL distribution. (I) $(\lambda=3, \gamma=3, \beta=3, \delta=2, \theta=2), \quad$ (II) $(\lambda=2, \gamma=2.5, \beta=1.5, \delta=1, \theta=2.5)$, 


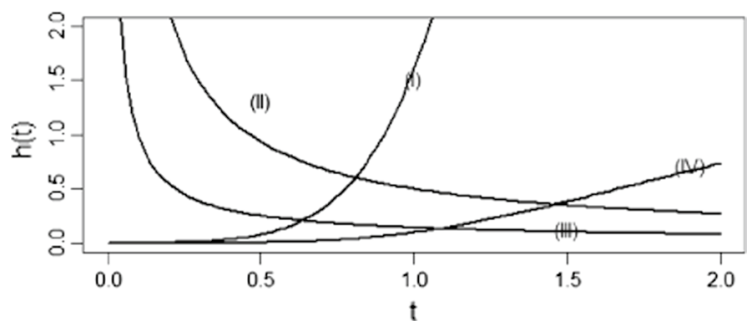

Fig (2.I): $(\lambda=1, \gamma=0.5, \beta=1, \delta=0.1, \theta=0.2), \quad$ Fig (2.II): $(\lambda=0.25, \gamma=0.25, \beta=1.75, \delta=0.2, \theta=1.5)$, Fig (2.III): $(\lambda=0.1, \gamma=2, \beta=3, \delta=2, \theta=1), \quad \operatorname{Fig}(2 . \mathrm{IV}):(\lambda=2, \gamma=3, \beta=0.9, \delta=1.5, \theta=3)$,

Fig. $2 \mathrm{~h}(\mathrm{t})$ of the POGEL distribution. (I) $(\lambda=1, \gamma=0.5, \beta=1, \delta=0.1, \theta=0.2), \quad$ (II) $(\lambda=0.25, \gamma=0.25, \beta=1.75, \delta=0.2, \theta=1.5), \quad($ III $) \quad(\lambda=0.1, \gamma=2, \beta=3, \delta=2, \theta=1), \quad$ (IV) $(\lambda=2, \gamma=3, \beta=0.9, \delta=1.5, \theta=3)$

\section{Graphical description}

The pdf curves of POGEL distribution are plotted in Fig. 1 for some selected values of the parameters. $(\lambda=3, \gamma=3, \beta=3, \delta=2, \theta=2)$, $(\lambda=2, \gamma=2.5, \beta=1.5, \delta=1, \theta=2.5),(\lambda=0.3, \gamma=0.25, \beta=1.2, \delta=1, \theta=1.5)$, $(\lambda=1.5, \gamma=1.5, \beta=2, \delta=3, \theta=1.5)$.

Figure 1 shows that:

- The $f(t)$ curves of the POGEL are more flexible for changing values of the parameters.

- The $\mathrm{f}(\mathrm{t})$ curves take various shapes such as symmetrical, right-skewed, reversed J-shaped and unimodal.

The $h(t)$ curves of two POGEL populations are plotted in Fig. 2. The first population is when $\gamma<1,(\lambda=0.25, \gamma=0.25, \beta=2, \delta=1, \theta=1.5)$, $(\lambda=0.25, \gamma=0.25, \beta=1.2, \delta=1, \theta=1.5)$, The second population is when $\gamma>1$, $(\lambda=2, \gamma=3, \beta=1.3, \delta=2, \theta=3),(\lambda=2, \gamma=3, \beta=0.9, \delta=1.5, \theta=3)$.

Figure 2 show that:

- The $h(t)$ curves of the POGEL are more flexible for changing values of the parameters.

- The $\mathrm{h}(\mathrm{t})$ curves take different shapes such as constant, increasing, decreasing, and reversed $\mathrm{J}$ shape.

This fact implies that the POGEL can be very useful for fitting data sets with various shapes. 


\section{Some Special Cases}

The importance of POGEL distribution is that it contains several special cases (sub-models), by using (3) as follows:

(I) Odd generalized exponential Lomax distribution

The odd generalized exponential Lomax (OGEL) introduced by [19] is a special case from POGEL distribution, when $\delta=1$ which is given in Eq. (1).

(II) Odds generalized exponential power Lomax distribution

The odds generalized exponential power Lomax distribution (OGEPL) introduced by [21] when $\gamma=1 \mathrm{in}$ (3) is a special case from POGEL with the following pdf:

$f(t ; \lambda, \beta, \delta, \theta)=\frac{\beta \delta \lambda}{\theta} t^{\delta-1}\left(1+\frac{t^{\delta}}{\theta}\right)^{\beta-1} e^{-\lambda\left[\left(1+\frac{t^{\delta}}{\theta}\right)^{\beta}-1\right]}, t>0, \lambda, \beta, \delta>0, \theta>1$.

(III) Odd exponential Lomax distribution

Odd exponential Lomax distribution (OEL) introduced by [9] when $\gamma=\delta=1$ in (3) is a special case from POGEL with the following pdf:

$$
f(t ; \lambda, \beta, \theta)=\frac{\beta \lambda}{\theta}\left(1+\frac{t}{\theta}\right)^{\beta-1} e^{-\lambda\left[\left(1+\frac{t}{\theta}\right)^{\beta}-1\right]}, t>0, \lambda, \beta>0, \theta>1 .
$$

(IV) Exponential distribution

The exponential distribution (E) with two parameters introduced by [15] when $\gamma=\delta=\beta=1$ in (3) is a special case from POGEL with the following pdf:

$$
f(t ; \lambda, \theta)=\frac{\lambda}{\theta} e^{-\frac{\lambda}{\theta} t}, t>0, \lambda>0, \theta>1 .
$$

(V) Generalized exponential distribution

The generalized exponential distribution (GE) introduced by [16] when $\mathrm{y}=\left[\left(1+\frac{\mathrm{t}^{\delta}}{\theta}\right)^{\beta}-1\right]$ in (3) is a special case from POGEL with the following pdf:

$$
f(y ; \lambda, \gamma)=\lambda \gamma e^{-\lambda y}\left[1-e^{-\lambda y}\right]^{\gamma-1}, y>0, \lambda, \gamma>0 \text {. }
$$

\section{Parameter Estimation}

In this Section, the ML and the MPS methods are discussed to obtain the estimator of parameters of the POGEL under complete samples. 


\subsection{Maximum Likelihood Estimation}

The ML is used to estimate the unknown parameters of the POGEL distribution based on complete samples [22].

Let $t_{1}, \ldots, t_{n}$ be a random sample of size $\mathrm{n}$ from POGEL, with parameters $\underline{\omega}=(\lambda, \gamma, \beta, \delta, \theta)$, the likelihood function of the density is given by,

$$
L(\underline{\omega} ; \underline{t})=\left(\frac{\lambda \gamma \beta \delta}{\theta}\right)^{n} \prod_{i=1}^{n}\left(1+\frac{t_{i}^{\delta}}{\theta}\right)^{\beta-1} t_{i}^{\delta-1} e^{-\lambda\left[\left(1+\frac{t_{i}^{\delta}}{\theta}\right)^{\beta}-1\right]}\left\{1-e^{-\lambda\left[\left(1+\frac{t_{i}^{\delta}}{\theta}\right)^{\beta}-1\right]}\right\}^{\gamma-1}
$$

Then, the log likelihood function, denoted Lne

$$
\begin{aligned}
\operatorname{Ln} \ell= & n \operatorname{Ln}(\lambda)+n \operatorname{Ln}(\gamma)+n \operatorname{Ln}(\beta)+n \ln (\delta)-n \operatorname{Ln}(\theta)+(\delta-1) \sum_{i=1}^{n} \ln \left(t_{i}\right) \\
& +(\beta-1) \sum_{i=1}^{n} \operatorname{Ln}\left(1+\frac{t_{i}^{\delta}}{\theta}\right)-\lambda \sum_{i=1}^{n}\left[\left(1+\frac{t_{i}^{\delta}}{\theta}\right)^{\beta}-1\right] \\
& +(\gamma-1) \sum_{i=1}^{n} \operatorname{Ln}\left\{1-e^{-\lambda\left[\left(1+\frac{t_{i}^{\delta}}{\theta}\right)^{\beta}-1\right]}\right.
\end{aligned}
$$

The log-likelihood (30) can be maximized numerically using the R (optim function), for interval estimation of the model parameters, it requires the $4 \times 4$ observed information matrix $\mathrm{I}(\omega)=\{\underline{\omega}\}$ for $\underline{\omega}=(\lambda, \gamma, \beta, \delta, \theta)$. Under standard regularity conditions, the multivariate normal $\mathrm{N}_{\mathrm{m}}^{-}(\omega)\left(0,(\omega)^{-1}\right)$ distribution can be used to construct approximate confidence intervals for the parameters. Here, $\mathrm{I}(\hat{\omega})$ is the total observed information matrix evaluated at $\hat{\omega}$. [see "Appendix A"].

\subsection{Maximum Product of Spacing Estimation Method}

One of the most common methods for estimating the parameters of a distribution is the ML method. Although this method is consistent, asymptotically efficient, it was found to be unbounded and inefficient in the estimation in various cases, such as involving certain mixtures of continuous distributions, heavy-tailed distributions and J-shaped distributions [23].

The MPS method was introduced by [24] as an alternative to ML for the estimation of parameters of continuous univariate distributions. The MPS estimators are consistent, asymptotically normal and efficient.

Suppose that an ordered random sample $t_{1}, \ldots, t_{n}$ drawn from POGEL distribution with parameters $\underline{\omega}=(\lambda, \gamma, \beta, \delta, \theta)$ and cdf (8) the spacing is constructed as: 


$$
D_{i}(\omega)=F_{\omega}\left(t_{i: n}\right)-F_{\omega}\left(t_{i-1: n}\right), i=1, \ldots, n .
$$

where $\sum_{i=1}^{n} D_{i}=1$.

To estimate the unknown parameters, the product spacings is defined and the geometric mean of spacings is maximized as follows:

$$
G=\left[\prod_{i=1}^{n+1} D_{i}(\omega)\right]^{\frac{1}{n+1}}=\left\{\prod_{i=1}^{n+1}\left[F_{\omega}\left(t_{i: n}\right)-F_{\omega}\left(t_{i-1: n}\right)\right]\right\}^{\frac{1}{n+1}} .
$$

Then, by taking the logarithm of G:

$$
\ln G=\frac{1}{n+1} \sum_{i=1}^{n+1} \ln \left\{F_{\omega}\left(t_{i: n}\right)-F_{\omega}\left(t_{i-1: n}\right\}, \operatorname{let} H=\ln G\right.
$$

In this study the maximization of the quantity in (33) is defined as:

$$
\hat{\omega}_{M P S}=\arg \max \sum_{i=1}^{n+1} \ln \left\{F_{\omega}\left(t_{i: n}\right)-F_{\omega}\left(t_{i-1: n}\right)\right\}
$$

Substitute (4) in (33) the function $\mathrm{H}$ is given by:

$$
\begin{aligned}
& H=\frac{1}{n+1}\left\{\ln D_{1}+\sum_{i=2}^{n} \ln D_{i}+\ln D_{n+1}\right\} \\
& =\frac{1}{n+1}\left\{\ln \left[1-e^{-\lambda\left[\left(1+\frac{t_{i}^{\delta}}{\theta}\right)^{\beta}-1\right]}\right]^{\gamma}+\sum_{i=2}^{n} \ln \left[\left[1-e^{-\lambda\left[\left(1+\frac{t_{i}^{\delta}}{\theta}\right)^{\beta}-1\right]}\right]^{\gamma}\right.\right. \\
& \left.\left.-\left[1-e^{-\lambda\left[\left(1+\frac{t_{(i-1)}^{\delta}}{\theta}\right)^{\beta}-1\right]}\right]^{\gamma}\right]\right\}+\frac{1}{n+1}\left\{\ln \left[1-e^{-\lambda\left[\left(1+\frac{t_{n}^{\delta}}{\theta}\right)^{\beta}-1\right]}\right]^{\gamma}\right\}
\end{aligned}
$$

Taking the partial derivative of (35) with respect to $\underline{\omega}=(\lambda, \gamma, \beta, \delta, \theta)$ and equating to zero

$$
\begin{aligned}
& \frac{\partial}{\partial \lambda} H(\underline{\omega})=\frac{1}{n+1} \sum_{i=1}^{n+1} \frac{1}{D i(\underline{\omega})}\left[\Delta_{1}\left(t_{i, n} \mid \underline{\omega}\right)-\Delta_{1}\left(t_{i-1, n} \mid \underline{\omega}\right)\right]=0 \\
& \frac{\partial}{\partial \gamma} H(\underline{\omega})=\frac{1}{n+1} \sum_{i=1}^{n+1} \frac{1}{D i(\underline{\omega})}\left[\Delta_{2}\left(t_{i, n} \mid \underline{\omega}\right)-\Delta_{2}\left(t_{i-1, n} \mid \underline{\omega}\right)\right]=0
\end{aligned}
$$




$$
\begin{aligned}
& \frac{\partial}{\partial \beta} H(\underline{\omega})=\frac{1}{n+1} \sum_{i=1}^{n+1} \frac{1}{D i(\underline{\omega})}\left[\Delta_{3}\left(t_{i, n} \mid \underline{\omega}\right)-\Delta_{3}\left(t_{i-1, n} \mid \underline{\omega}\right)\right]=0 \\
& \frac{\partial}{\partial \delta} H(\underline{\omega})=\frac{1}{n+1} \sum_{i=1}^{n+1} \frac{1}{D i(\underline{\omega})}\left[\Delta_{4}\left(t_{i, n} \mid \underline{\omega}\right)-\Delta_{4}\left(t_{i-1, n} \mid \underline{\omega}\right)\right]=0 \\
& \frac{\partial}{\partial \theta} H(\underline{\omega})=\frac{1}{n+1} \sum_{i=1}^{n+1} \frac{1}{D i(\underline{\omega})}\left[\Delta_{5}\left(t_{i, n} \mid \underline{\omega}\right)-\Delta_{5}\left(t_{i-1, n} \mid \underline{\omega}\right)\right]=0
\end{aligned}
$$

where,

$$
\begin{aligned}
& \Delta_{1}=\left(t_{i, n} \mid \omega\right)=-e^{-\lambda\left[\left(1+\frac{t^{\delta}}{\theta}\right)^{\beta}-1\right]} \gamma\left[-\left(\frac{t^{\delta}}{\theta}+1\right)^{\beta}+1\right]\left[1-e^{-\lambda\left[\left(1+\frac{t^{\delta}}{\theta}\right)^{\beta}-1\right]}\right]^{\gamma-1} \\
& \Delta_{2}=\left(t_{i, n} \mid \omega\right)=\left[1-e^{-\lambda\left[\left(1+\frac{t^{\delta}}{\theta}\right)^{\beta}-1\right]}\right]^{\gamma} \operatorname{Ln}\left\{1-e^{-\lambda\left[\left(1+\frac{t^{\delta}}{\theta}\right)^{\beta}-1\right]}\right\} \\
& \Delta_{3}=\left(t_{i, n} \mid \omega\right)=\gamma\left[1-e^{-\lambda\left[\left(1+\frac{t^{\delta}}{\theta}\right)^{\beta}-1\right]}\right]^{\gamma-1} \lambda e^{-\lambda\left[\left(1+\frac{t^{\delta}}{\theta}\right)^{\beta}-1\right]} \operatorname{Ln}\left(\frac{t^{\delta}}{\theta}+1\right)\left(\frac{t^{\delta}}{\theta}+1\right)^{\beta} \\
& \Delta_{4}=\left(t_{i, n} \mid \omega\right)=-\gamma\left[1-e^{-\lambda\left[\left(1+\frac{t^{\delta}}{\theta}\right)^{\beta}-1\right]}\right]^{\gamma-1} e^{-\lambda\left[\left(1+\frac{t^{\delta}}{\theta}\right)^{\beta}-1\right]} \ln (t) t^{\delta} \\
& \Delta_{5}=\left(t_{i, n} \mid \omega\right)=\frac{\gamma \beta \delta \lambda}{\theta^{2}} t^{\delta}\left(1+\frac{t^{\delta}}{\theta}\right)^{\beta-1} e^{-\lambda\left[\left(1+\frac{t^{\delta}}{\theta}\right)^{\beta}-1\right]}\left[1-e^{-\lambda\left[\left(1+\frac{t^{\delta}}{\theta}\right)^{\beta}-1\right]}\right]^{\gamma-1}
\end{aligned}
$$

[23] Showed that maximizing $H$ as a method of parameter estimation is as efficient as ML estimation, and the MPS estimators are consistent under more general conditions than the ML estimators.

The MPS method shows asymptotic properties like the ML estimators [23]. Introduced the variance covariance matrix of the MPS estimators. Therefore, the asymptotic properties of MPS can be used to construct the asymptotic confidence intervals for the parameters [25]. Let $I\left(\begin{array}{c}\hat{\omega} \\ -\end{array}\right)$ is the observed Fishers information matrix it can be defined as: 


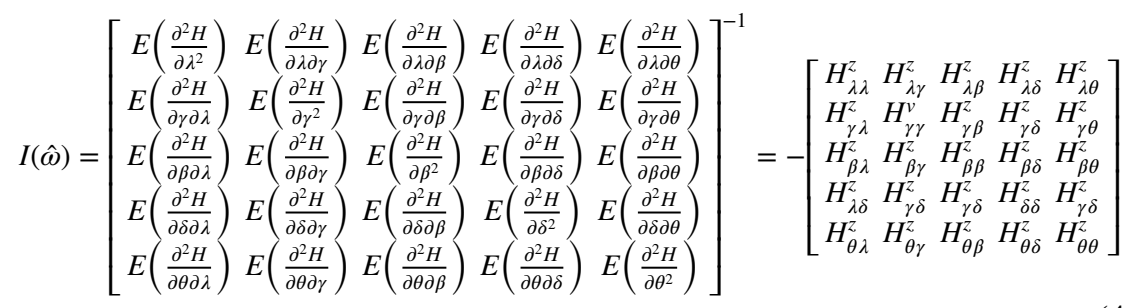

So on the basis of these derivatives the information matrix $I(\underline{\hat{\omega}})$ can be obtained. The approximate $(1-b) 100 \%$ confidence intervals for the parameters $\lambda, \gamma, \beta, \delta$ and $\theta$ are given.

\section{Simulation Study}

In this Section a simulation study is introduced to illustrate the theoretical results considering ML and MPS methods on the basis of generated data from POGEL distribution by taking the parameter $\theta$ as known for all methods of estimation.

For each method of estimation, initial parameter values and sample sizes, the estimates, mean square error (MSE), relative bias (RB) and asymptotic confidence intervals (ACI) are calculated using the following formulae:

(1) Bias $^{2}=(\text { estimate of the parameter }- \text { true value of the parameter })^{2}$

(2) $M S E=$ mean $\left[(\text { estimator }- \text { true value })^{2}\right]$.

(3) $R B=\frac{\text { Bias }}{\text { true value }}$.

(4) The ACI of the ML estimation for the multivariate normal of the parameters $\widehat{\omega}=(\hat{\lambda}, \hat{\gamma}, \widehat{\beta}, \hat{\delta}, \theta)$ can be used to compute the asymptotic $100(1-\vartheta) \%, 0<\vartheta<1$, for the parameters as follows:

$$
\hat{\omega} \pm z_{\left(1-\frac{\vartheta}{2}\right)} \sqrt{\operatorname{Var}(\hat{\lambda}, \hat{\gamma}, \hat{\beta}, \hat{\delta})}
$$

(5) The ACI of MPS estimation for the parameters $\hat{\omega}=(\hat{\lambda}, \hat{\gamma}, \hat{\beta}, \hat{\delta}, \theta)$ can be used to compute the asymptotic $100(1-b) \%, 0<b<1$, for the parameters as follows

$$
\hat{v} \pm \tau_{\left(\frac{b}{2}\right)} \sqrt{\operatorname{Var}(\hat{\lambda}, \hat{\gamma}, \hat{\beta}, \hat{\delta})} .
$$

where $\tau_{\left(\frac{b}{2}\right)}$ is the upper $\left(\frac{b}{2}\right)$ percentile of the standard normal distribution. 
Table 1 The Estimates, MSEs, RB and ACI for MLE and MPS for the parameters $(\lambda=0.5$, $\gamma=1.5, \beta=1.5, \delta=1.5, \theta=1.5$ ) for POGEL distribution for 1000 repetitions and different sample sizes

\begin{tabular}{|c|c|c|c|c|c|c|c|}
\hline \multirow[t]{2}{*}{$\mathrm{n}$} & \multirow[t]{2}{*}{ Methods } & \multirow[t]{2}{*}{ Parameters } & \multirow[t]{2}{*}{ Estimates } & \multirow[t]{2}{*}{ MSE } & \multirow[t]{2}{*}{$\mathrm{RB}$} & \multicolumn{2}{|l|}{ ACI } \\
\hline & & & & & & LI & UI \\
\hline \multirow[t]{8}{*}{50} & \multirow[t]{4}{*}{ ML } & $\lambda$ & 1.09 & 5.65 & 1.19 & 0.05 & 5.03 \\
\hline & & $\gamma$ & 2.24 & 8.75 & 0.49 & 0.39 & 8.85 \\
\hline & & B & 1.25 & 0.73 & 0.16 & 0.25 & 3.61 \\
\hline & & $\delta$ & 1.77 & 1.30 & 0.18 & 0.63 & 4.84 \\
\hline & \multirow[t]{4}{*}{ MPS } & $\lambda$ & 0.78 & 0.64 & 0.30 & 0.04 & 5.71 \\
\hline & & $\gamma$ & 1.71 & 2.02 & 0.14 & 0.54 & 4.17 \\
\hline & & $\beta$ & 1.41 & 1.65 & 1.82 & 0.20 & 4.79 \\
\hline & & $\delta$ & 1.60 & 1.37 & 0.07 & 0.54 & 4.51 \\
\hline \multirow[t]{8}{*}{100} & \multirow[t]{4}{*}{ ML } & $\lambda$ & 0.74 & 0.43 & 0.47 & 0.09 & 2.24 \\
\hline & & $\gamma$ & 1.84 & 1.51 & 0.22 & 0.50 & 4.83 \\
\hline & & $\beta$ & 1.29 & 0.66 & 0.14 & 0.37 & 3.50 \\
\hline & & $\delta$ & 1.66 & 0.98 & 0.10 & 0.65 & 4.22 \\
\hline & \multirow[t]{4}{*}{ MPS } & $\lambda$ & 0.77 & 0.60 & 0.55 & 0.01 & 2.64 \\
\hline & & $\gamma$ & 1.44 & 0.61 & 0.04 & 0.55 & 3.41 \\
\hline & & $\beta$ & 1.56 & 1.30 & 0.04 & 0.31 & 4.40 \\
\hline & & $\delta$ & 1.75 & 0.76 & 0.17 & 0.61 & 3.72 \\
\hline \multirow[t]{8}{*}{150} & \multirow[t]{4}{*}{ ML } & $\lambda$ & 0.73 & 0.28 & 0.45 & 0.14 & 1.84 \\
\hline & & $\gamma$ & 1.82 & 0.88 & 0.21 & 0.59 & 3.85 \\
\hline & & $\beta$ & 1.29 & 0.54 & 0.13 & 0.37 & 3.27 \\
\hline & & $\delta$ & 1.54 & 0.65 & 0.03 & 0.66 & 3.53 \\
\hline & \multirow[t]{4}{*}{ MPS } & $\lambda$ & 0.73 & 0.41 & 0.46 & 0.10 & 2.17 \\
\hline & & $\gamma$ & 1.47 & 0.59 & 0.02 & 0.59 & 3.40 \\
\hline & & $\beta$ & 1.47 & 1.03 & 0.02 & 0.36 & 3.96 \\
\hline & & $\delta$ & 1.71 & 0.56 & 0.14 & 0.57 & 3.30 \\
\hline \multirow[t]{8}{*}{200} & \multirow[t]{4}{*}{ ML } & $\lambda$ & 0.72 & 0.19 & 0.43 & 0.16 & 1.52 \\
\hline & & $\gamma$ & 1.73 & 0.54 & 0.15 & 0.61 & 3.33 \\
\hline & & $\beta$ & 1.24 & 0.49 & 0.17 & 0.43 & 2.97 \\
\hline & & $\delta$ & 1.53 & 0.50 & 0.02 & 0.75 & 3.48 \\
\hline & \multirow[t]{4}{*}{ MPS } & $\lambda$ & 0.72 & 0.25 & 0.46 & 0.12 & 1.82 \\
\hline & & $\gamma$ & 1.47 & 0.47 & 0.02 & 0.59 & 3.25 \\
\hline & & $\beta$ & 1.38 & 0.83 & 0.07 & 0.38 & 3.77 \\
\hline & & $\delta$ & 1.70 & 0.42 & 0.14 & 0.67 & 3.04 \\
\hline \multirow[t]{8}{*}{300} & \multirow[t]{4}{*}{ ML } & $\lambda$ & 0.67 & 0.11 & 0.35 & 0.18 & 1.30 \\
\hline & & $\gamma$ & 1.74 & 0.41 & 0.16 & 0.67 & 3.30 \\
\hline & & $\beta$ & 1.30 & 0.46 & 0.13 & 0.47 & 3.21 \\
\hline & & $\delta$ & 1.45 & 0.40 & 0.03 & 0.68 & 3.14 \\
\hline & \multirow[t]{4}{*}{ MPS } & $\lambda$ & 0.69 & 0.16 & 0.38 & 0.14 & 1.49 \\
\hline & & $\Gamma$ & 1.39 & 0.36 & 0.07 & 0.62 & 2.92 \\
\hline & & $\beta$ & 1.41 & 0.71 & 0.05 & 0.45 & 3.69 \\
\hline & & $\delta$ & 1.75 & 0.35 & 0.16 & 0.77 & 2.88 \\
\hline
\end{tabular}


Table 2 The Estimates, MSEs, RBs and ACI for MLE and MPS for the parameters $(\lambda=0.8$, $\gamma=1.8, \beta=1.2, \delta=1.5, \theta=1.5$ ) for POGEL distribution for 1000 repetitions and different sample sizes

\begin{tabular}{|c|c|c|c|c|c|c|c|}
\hline \multirow[t]{2}{*}{$\mathrm{n}$} & \multirow[t]{2}{*}{ Methods } & \multirow[t]{2}{*}{ Parameters } & \multirow[t]{2}{*}{ Estimates } & \multirow[t]{2}{*}{ MSE } & \multirow[t]{2}{*}{$\mathrm{RB}$} & \multicolumn{2}{|l|}{ ACI } \\
\hline & & & & & & LI & UI \\
\hline \multirow[t]{8}{*}{50} & \multirow[t]{4}{*}{ ML } & $\lambda$ & 1.47 & 14.03 & 0.84 & 0.03 & 6.12 \\
\hline & & $\gamma$ & 2.95 & 20.77 & 0.64 & 0.33 & 11.37 \\
\hline & & $\beta$ & 1.24 & 0.66 & 0.03 & 0.20 & 3.54 \\
\hline & & $\delta$ & 1.91 & 2.15 & 0.28 & 0.66 & 6.02 \\
\hline & \multirow[t]{4}{*}{ MPS } & $\lambda$ & 1.99 & 22.65 & 1.37 & 0.05 & 9.37 \\
\hline & & $\gamma$ & 2.68 & 53.64 & 0.49 & 0.42 & 10.11 \\
\hline & & $\beta$ & 1.26 & 1.23 & 0.05 & 0.15 & 4.25 \\
\hline & & $\delta$ & 1.84 & 1.64 & 0.23 & 0.61 & 4.85 \\
\hline \multirow[t]{8}{*}{100} & \multirow[t]{4}{*}{ ML } & $\lambda$ & 1.06 & 1.53 & 0.33 & 0.11 & 3.77 \\
\hline & & $\gamma$ & 2.25 & 3.26 & 0.25 & 0.46 & 5.94 \\
\hline & & $\beta$ & 1.18 & 0.42 & 0.02 & 0.27 & 3.02 \\
\hline & & $\delta$ & 1.77 & 1.27 & 0.18 & 0.74 & 4.67 \\
\hline & \multirow[t]{4}{*}{ MPS } & $\lambda$ & 1.06 & 1.24 & 0.32 & 0.12 & 3.81 \\
\hline & & $\gamma$ & 1.88 & 1.83 & 0.04 & 0.56 & 5.10 \\
\hline & & $\beta$ & 1.22 & 0.77 & 0.02 & 0.25 & 3.62 \\
\hline & & $\delta$ & 1.78 & 1.05 & 0.03 & 0.66 & 4.20 \\
\hline \multirow[t]{8}{*}{150} & \multirow[t]{4}{*}{ ML } & $\lambda$ & 0.90 & 0.43 & 0.14 & 0.16 & 2.30 \\
\hline & & $\gamma$ & 2.09 & 1.79 & 0.16 & 0.54 & 5.30 \\
\hline & & $\beta$ & 1.17 & 0.34 & 0.02 & 0.34 & 2.80 \\
\hline & & $\delta$ & 1.72 & 0.99 & 0.15 & 0.77 & 4.38 \\
\hline & \multirow[t]{4}{*}{ MPS } & $\lambda$ & 0.97 & 0.42 & 0.12 & 0.16 & 2.81 \\
\hline & & $\gamma$ & 1.85 & 1.57 & 0.03 & 0.58 & 4.27 \\
\hline & & $\beta$ & 1.17 & 0.33 & 0.02 & 0.31 & 2.78 \\
\hline & & $\delta$ & 1.77 & 0.92 & 0.14 & 0.69 & 4.08 \\
\hline \multirow[t]{8}{*}{200} & \multirow[t]{4}{*}{ ML } & $\lambda$ & 0.90 & 0.27 & 0.13 & 0.22 & 2.05 \\
\hline & & $\gamma$ & 2.05 & 1.40 & 0.14 & 0.58 & 4.26 \\
\hline & & $\beta$ & 1.16 & 0.27 & 0.03 & 0.30 & 2.40 \\
\hline & & $\delta$ & 1.65 & 0.72 & 0.10 & 0.68 & 3.84 \\
\hline & \multirow[t]{4}{*}{ MPS } & $\lambda$ & 0.95 & 0.33 & 0.11 & 0.20 & 2.03 \\
\hline & & $\gamma$ & 1.76 & 0.65 & 0.01 & 0.56 & 3.77 \\
\hline & & $\beta$ & 1.09 & 0.25 & 0.01 & 0.28 & 2.35 \\
\hline & & $\delta$ & 1.77 & 0.70 & 0.09 & 0.66 & 3.77 \\
\hline \multirow[t]{8}{*}{300} & \multirow[t]{4}{*}{ ML } & $\lambda$ & 0.89 & 0.14 & 0.12 & 0.21 & 1.78 \\
\hline & & $\gamma$ & 2.03 & 0.87 & 0.13 & 0.56 & 4.12 \\
\hline & & $\beta$ & 1.14 & 0.21 & 0.04 & 0.29 & 2.32 \\
\hline & & $\delta$ & 1.59 & 0.52 & 0.06 & 0.64 & 3.57 \\
\hline & \multirow[t]{4}{*}{ MPS } & $\lambda$ & 0.91 & 0.12 & 0.11 & 0.19 & 1.74 \\
\hline & & $\gamma$ & 1.74 & 0.51 & 0.04 & 0.53 & 3.43 \\
\hline & & $\beta$ & 1.04 & 0.19 & 0.02 & 0.26 & 2.09 \\
\hline & & $\delta$ & 1.76 & 0.50 & 0.04 & 0.61 & 3.52 \\
\hline
\end{tabular}


Table 3 Descriptive Statistics for the COVID-19 data set

\begin{tabular}{lllllllll}
\hline Mean & Median & Mode & St.D & Variance & Skewness & Kurtosis & 25th P & 75th P \\
\hline 411.9 & 397.5 & 402 & 417.0 & $173,900.7$ & 1.24 & 0.97 & 59.5 & 550.8 \\
\hline
\end{tabular}

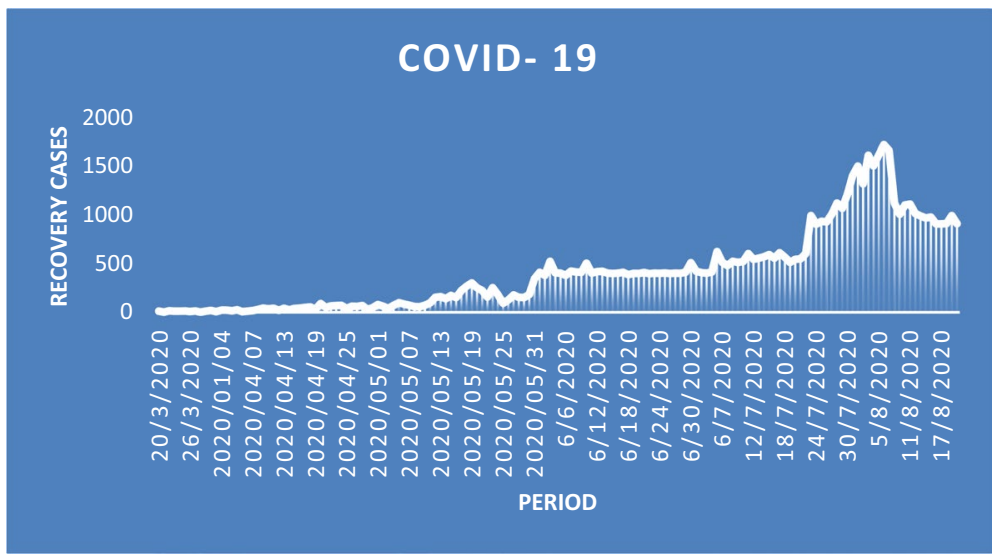

Fig. 3 The recovery cases for the COVID-19 data set

The following steps are used to compute the ML and MPS estimates for POGEL distribution for different sample sizes [ $\mathrm{n}=50,100,150,200,300]$.

1. Generate random samples of size $\mathrm{n}$ from POGEL distribution $[\mathrm{n}=50,100,150$, 200,300] by using (9).

2. Obtain the ML estimates.

3. Obtain the MPS estimates by solving Eqs. (36-40).

4. Compute the MSE, RB and ACI for each estimate and for the ML and MPS methods using Eqs. (47), (48), (49) and (50).

5. Repeat the above steps for all methods of estimation and different sample sizes with 1000 repetitions.

All the above steps 1-5 are calculating using $\mathrm{R}$ Studio program version (1.3.1073).

The results of the simulation study are illustrated in Tables 1 and 2. From these tables, it is noticeable that:

- As expected the MSE, RB and ACI decreased when $n$ increased.

- The MSE of the MPS estimates is less than the MSE of the ML estimates for all parameters and sample sizes except for the parameters $\beta$ and $\delta$ at $n=50$ in Table 1 and for the parameters $\lambda$ and $\beta$ at $n=50$ in Table 2 . 

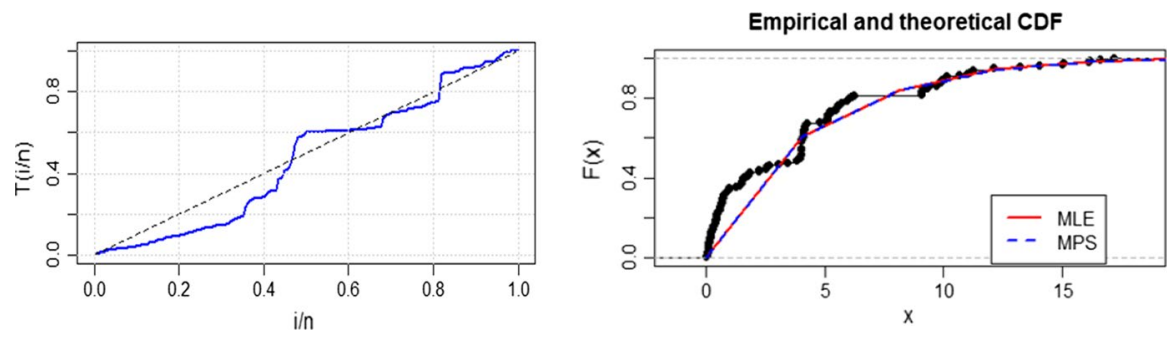

Fig. 4 The TTT plot and the Empirical cdf of the POGEL distribution for the COVID-19 data set

- The RB of the MPS estimates is less than the RB of the ML estimates for all parameters and sample sizes except for the parameters $\beta$ at $n=50$ in Table 1 and for the parameter $\lambda$ at $n=50$ in Table 2 .

- As expected the performance of the MPS estimates is appropriate than the ML estimates.

\section{Data Analysis}

In this Section, the COVID-19 data set is analyzed to illustrate the flexibility of POGEL distribution. The data set is taken from the MINISTRY of HEALTH reports in EGYPT referred to daily recovery cases of a random sample of 154 days in the interval of (20 March to 20 August 2020) of coronavirus patients in the ARAB REPUBLIC of EGYPT these data are given as.

112151212139145111872222152561217294336422141263943 $50553189496569713962606831467962407098 \quad 857258577397154$ 16014017315122226830225222315725417993127178154152182344 410380523406402380423414411503402417421402398401411387400

Table 4 The ML and MPS Estimates, SEs, ACI of the model parameters $(\lambda, \gamma, \beta, \delta, \theta)$ for the 154 daily recovery cases (in months) of COVID- 19 patient's data

\begin{tabular}{|c|c|c|c|c|c|c|}
\hline \multirow[t]{2}{*}{ Methods } & \multirow[t]{2}{*}{ Parameters } & \multirow[t]{2}{*}{ Estimates } & \multirow[t]{2}{*}{ SEs } & \multicolumn{2}{|l|}{ ACI } & \multirow[t]{2}{*}{ KS } \\
\hline & & & & LI & UI & \\
\hline \multirow[t]{5}{*}{ ML } & $\lambda$ & 1.06 & 1.53 & 0.81 & 1.30 & \multirow[t]{5}{*}{0.67} \\
\hline & $\gamma$ & 0.61 & 0.36 & 0.54 & 0.66 & \\
\hline & $\beta$ & 2.41 & 2.75 & 1.98 & 2.85 & \\
\hline & $\delta$ & 1.05 & 0.56 & 21.86 & 22.04 & \\
\hline & $\theta$ & 21.95 & 57.91 & 12.81 & 31.10 & \\
\hline \multirow[t]{5}{*}{ MPs } & $\lambda$ & 0.43 & 0.88 & 0.28 & 0.56 & \multirow[t]{5}{*}{0.66} \\
\hline & $\gamma$ & 0.52 & 0.33 & 0.46 & 0.57 & \\
\hline & $\beta$ & 1.33 & 1.05 & 1.17 & 1.50 & \\
\hline & $\delta$ & 1.19 & 0.71 & 1.08 & 1.30 & \\
\hline & $\theta$ & 6.87 & 14.37 & 4.60 & 9.14 & \\
\hline
\end{tabular}




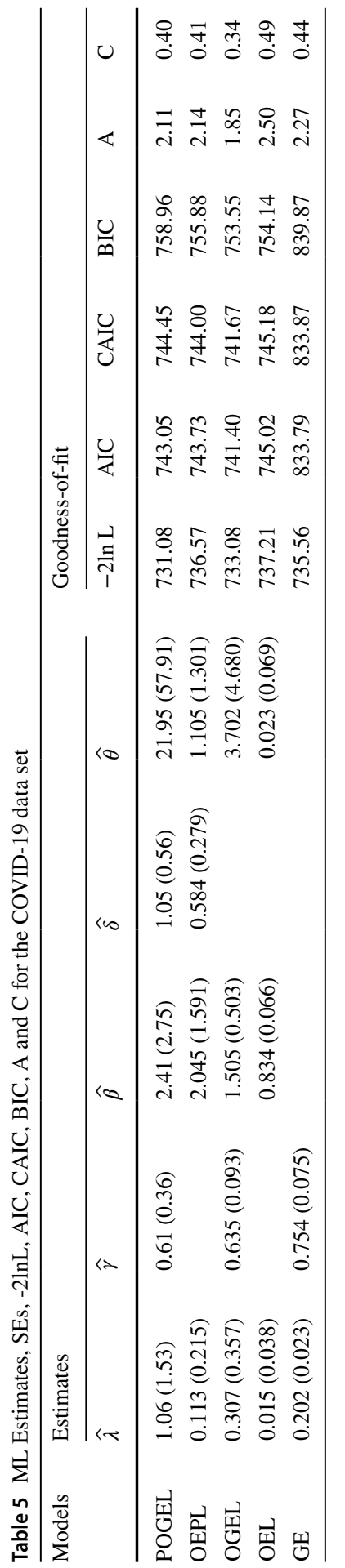



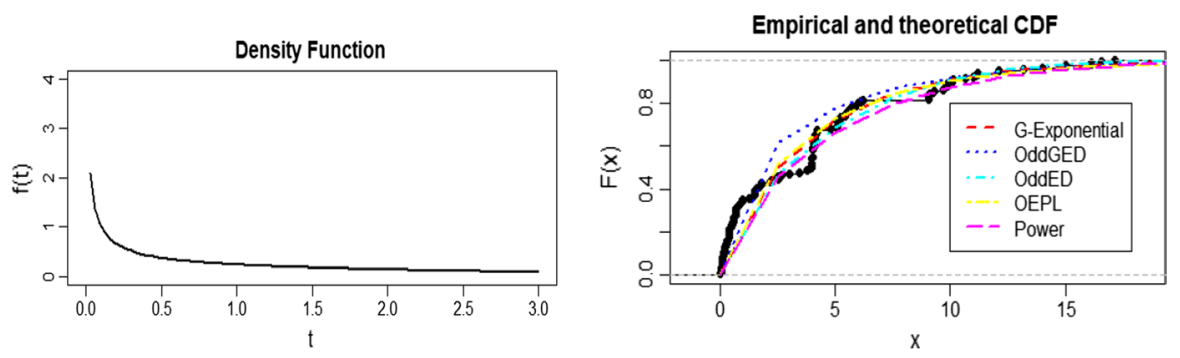

Fig. 5 The pdf with the value of the estimated parameters and cdfs of the POGEL model and other fitted models for the COVID-19 data set

\section{3 5125216025435565695915566115665125445496029919049339281007 112110661211140214991318161115031613171616551119100611011109 1013989968977908908911991909.}

It can be noticed from Table 3 that the data set is also right-skewed and platykurtic. Figure 3 presents the sequence time for the recovery cases of COVID-19.

Figure 3 show that.

- The curve take various shapes such as increasing, fixed and highly increasing.

- As expected increasing in injuries cases lead to increasing in recovery cases.

Before analyzing this data set, the scaled- TTT plot can be used to verify our distribution validity [26]. It allows identifying the shape of the $h(t)$ graphically. The empirical scaled-TTT plot of the COVID-19 data set is shown in Fig. 4. This Figure indicates that the TTT plot is convex then concave which indicates an upside down bathtub hazard rate. It verifies our distribution validity.

This data will be studied from two ways, firstly comparing the two methods of estimation. Secondly illustrate the importance and flexibility of the POGEL distribution with its sub-models [Generalized Exponential (GE), Odd Exponential Lomax.

(OEL), Odd Generalized Exponential Lomax (OGEL) and Odd Generalized Exponential Power Lomax (OGEPL) distributions)]. The plot of empirical cdf of the COVID-19 data set is displayed in Fig. 4 for the ML and MPS methods of estimations.

Table 4. Presents the parameter estimation for the ML and MPs methods with its standard errors (SEs).

Table 5 give the ML estimates and the corresponding standard errors (SEs) in parentheses of the parameters for all fitted models and the numerical values the Akaike information criterion (AIC), the consistent Akaike information criterion (CAIC), Bayesian Information Criterion (BIC), Anderson Darling (A) and Cramervon Mises (C) for POGEL and its sub models OEPL, OGEL, OEL and GE. For the COVID-19 data set, the -2ln L statistic for GE and OEL against POGEL is (4.48, 6.13 ) respectively. Therefore, there is a significant difference between GE, OEL and POGEL. Moreover, the values of the statistics AIC, CAIC and BIC are smaller for 
the POGEL distribution which mean that the POGEL distribution is a "appropriate" fit for the COVID-19 data, however a comparison of POGEL, OEPL and OGEL distributions shows that no significant difference between this distributions based on the values of AIC, CAIC and BIC which mean that the POGEL, OEPL and OGEL distributions are slightly appropriate for the COVID-19 data. The fitted cdfs for the COVID-19 data which supported this results are displayed in Fig. 5.

\section{Conclusions}

In this paper, Covid-19 studied statistically point of view depending on a probability distribution called Power Odd Generalized Exponential-Lomax distribution (POGEL). Some of its statistical properties were introduced. The estimation of the unknown model parameters was done with the maximum likelihood and maximum product spacing methods, with numerical guarantees on their behavior via a simulation study. The POGEL and some of its sub models are good fit for the ARAB REPUBLIC of EGYPT COVID- 19 data. From the estimated parameters of the POGEL it can predict that the curve of the daily recovery cases will be decreasing compared to daily cases which is consistent with the curve of the POGEL which takes the reversed J-shape. The POGEL distribution with an increasing but concave hazard rate best describes statistically how the cases respond to treatment. The concave shape is encouraging in the sense that the increase is happening at a decreasing rate. This points to a somewhat success in the interventions by government. The hazard rate is still increasing, and the number of recovery cases has not been stopped yet despite the number of the patients are on the way to decrease.

\section{Appendix A}

1. The first-order partial derivatives and the derivation of the elements of the fisher information matrix of ML

Calculating the first-order partial derivatives with respect to $\underline{\omega}=(\lambda, \gamma, \beta, \delta, \theta)$ and equating them to zero, we get the following nonlinear equations:

$$
\frac{\partial \operatorname{Ln} \ell}{\partial \lambda}=\frac{n}{\lambda}-\sum_{i=1}^{n}\left[\left(1+\frac{t_{i}^{\delta}}{\theta}\right)^{\beta}-1\right]+(\gamma-1) \sum_{i=1}^{n} \frac{e^{-\lambda\left[\left(1+\frac{t_{i}^{\delta}}{\theta}\right)^{\beta}-1\right]}\left[\left(1+\frac{t_{i}^{\delta}}{\theta}\right)^{\beta}-1\right]}{\left\{e^{\lambda\left[\left(1+\frac{t_{i}^{\delta}}{\theta}\right)^{\beta}-1\right]}-1\right\}}=0
$$




$$
\begin{aligned}
& \frac{\partial \operatorname{Ln} \ell}{\partial \gamma}=\frac{n}{\gamma}+\sum_{i=1}^{n} \operatorname{Ln}\left\{1-e^{-\lambda\left[\left(1+\frac{t_{i}^{\delta}}{\theta}\right)^{\beta}-1\right]}\right\}=0 \\
& \frac{\partial \operatorname{Ln} \ell}{\partial \beta}=\frac{n}{\beta}+\sum_{i=1}^{n} \operatorname{Ln}\left(1+\frac{t_{i}^{\delta}}{\theta}\right)-\lambda \sum_{i=1}^{n}\left\{\left(1+\frac{t_{i}^{\delta}}{\theta}\right)^{\beta} \operatorname{Ln}\left(1+\frac{t_{i}^{\delta}}{\theta}\right)\right\}+ \\
& (\gamma-1) \sum_{i=1}^{n} \frac{\lambda e^{-\frac{\lambda}{\theta^{\beta}}\left[\left(t_{i}^{\delta}+\theta\right)^{\beta}-\theta^{\beta}\right]}\left(1+\frac{t_{i}^{\delta}}{\theta}\right)^{\beta} \operatorname{Ln}\left(1+\frac{t_{i}^{\delta}}{\theta}\right)}{1-e^{-\lambda\left[\left(1+\frac{t_{i}^{\delta}}{\theta}\right)^{\beta}-1\right]}}=0 \\
& \frac{\partial \operatorname{Ln} \ell}{\partial \delta}=\frac{n}{\delta}+\sum_{i=1}^{n} \ln \left(t_{i}\right)+(\beta-1) \sum_{i=1}^{n} \frac{\ln \left(t_{i}\right) t_{i}^{\delta}}{1+\frac{t_{i}^{\delta}}{\theta}}-\lambda \sum_{i=1}^{n}\left\{\left(1+\frac{t_{i}^{\delta}}{\theta}\right)^{\beta-1} \beta t_{i}^{\delta} \operatorname{Ln}\left(t_{i}\right)\right\} \\
& +(\gamma-1) \sum_{i=1}^{n} \frac{\ln (t) t_{i}^{\delta}\left(1+\frac{t_{i}^{\delta}}{\theta}\right)^{\beta-1} \beta \lambda e^{-\lambda\left[\left(1+\frac{t_{i}^{\delta}}{\theta}\right)^{\beta}-1\right]}}{1-e^{-\lambda\left[\left(1+\frac{t_{i}^{\delta}}{\theta}\right)^{\beta}-1\right]}}=0 \\
& \frac{\partial \operatorname{Ln\ell }}{\partial \theta}=\frac{-n}{\theta}-(\beta-1) \sum_{i=1}^{n}\left(\frac{t_{i}^{\delta}}{\theta^{2}}\right)+\lambda \sum_{i=1}^{n}\left(\frac{t_{i}^{\delta}}{\theta^{2}}\right) \\
& +(\gamma-1) \sum_{i=1}^{n} \frac{\lambda \beta t_{i}^{\delta}\left(1+\frac{t_{i}^{\delta}}{\theta}\right)^{\beta-1} e^{-\lambda\left[\left(1+\frac{t_{i}^{\delta}}{\theta}\right)^{\beta}-1\right]}}{\theta^{2}\left\{1-e^{-\lambda\left[\left(1+\frac{t_{i}^{\delta}}{\theta}\right)^{\beta}-1\right]}\right\}}=0
\end{aligned}
$$

Taking the second partial derivatives for $I(\lambda, \gamma, \beta, \delta, \theta)$ as follows:

$$
\frac{\partial \ell^{2}}{\partial \lambda^{2}}=\frac{-n}{\lambda^{2}}-(\gamma-1) \sum_{i=1}^{n} \frac{\pi_{1}+\pi_{2}}{\left.\left[1-e^{-\lambda\left[\left(1+\frac{t_{i}^{\delta}}{\theta}\right)^{\beta}-1\right.}\right]\right]^{2}}=0
$$

where 


$$
\begin{gathered}
\pi_{1}=-\left[\left(1+\frac{t_{i}^{\delta}}{\theta}\right)^{\beta}-1\right]^{2} e^{-\lambda\left[\left(1+\frac{t_{i}^{\delta}}{\theta}\right)^{\beta}-1\right]}\left\{1-e^{-\lambda\left[\left(1+\frac{t_{i}^{\delta}}{\theta}\right)^{\beta}-1\right]}\right\} \\
\pi_{2}=\left[\left(1+\frac{t_{i}^{\delta}}{\theta}\right)^{\beta}-1\right]^{2} e^{-2 \lambda\left[\left(1+\frac{t_{i}^{\delta}}{\theta}\right)^{\beta}-1\right]} \\
\frac{\partial \ell^{2}}{\partial \lambda \partial \gamma}=\sum_{i=1}^{n} \frac{\left[\left(1+\frac{t_{i}^{\delta}}{\theta}\right)^{\beta}-1\right] e^{-2 \lambda\left[\left(1+\frac{t_{i}^{\delta}}{\theta}\right)^{\beta}-1\right]}}{\left\{1-e^{-\lambda\left[\left(1+\frac{t_{i}^{\delta}}{\theta}\right)^{\beta}-1\right]}\right\}}=0 \\
\frac{\partial \ell^{2}}{\partial \lambda \partial \beta}=-\sum_{i=1}^{n}\left(1+\frac{t_{i}^{\delta}}{\theta}\right)^{\beta} \ln \left(1+\frac{t_{i}^{\delta}}{\theta}\right)+(\gamma-1) \sum_{i=1}^{n} \frac{\pi_{3}+\pi_{4}}{\left[\begin{array}{l}
-\lambda\left[\left(1+\frac{t_{i}^{\delta}}{\theta}\right)^{\beta}-1\right. \\
\left.1-e^{2}\right]
\end{array}\right]^{2}=0}
\end{gathered}
$$

where

$$
\begin{aligned}
& \pi_{3}=\left(1+\frac{t_{i}^{\delta}}{\theta}\right)^{\beta}\left[\ln \left(1+\frac{t_{i}^{\delta}}{\theta}\right)\right] e^{-\lambda\left[\left(1+\frac{t_{i}^{\delta}}{\theta}\right)^{\beta}-1\right]}\left\{1-e^{-\lambda\left[\left(1+\frac{t_{i}^{\delta}}{\theta}\right)^{\beta}-1\right]}\right\} \\
& \left\{1-\lambda\left[\left(1+\frac{t_{i}^{\delta}}{\theta}\right)^{\beta}-1\right]\right\} \\
& \pi_{4}=\lambda\left[\left(1+\frac{t_{i}^{\delta}}{\theta}\right)^{\beta}-1\right]\left(1+\frac{t_{i}^{\delta}}{\theta}\right)^{\beta}\left[\ln \left(1+\frac{t_{i}^{\delta}}{\theta}\right)\right] e^{-2 \lambda\left[\left(1+\frac{t_{i}^{\delta}}{\theta}\right)^{\beta}-1\right]} \\
& \frac{\partial \ell^{2}}{\partial \lambda \partial \delta}=\sum_{i=1}^{n} \delta \beta\left(1+\frac{t_{i}^{\delta}}{\theta}\right)^{\beta-1} t_{i}^{\delta}\left[\ln \left(t_{i}\right)\right]+(\gamma-1) \sum_{i=1}^{n} \frac{\pi_{5}+\pi_{6}}{\left.\left[1-e^{-\lambda\left[\left(1+\frac{t_{i}^{\delta}}{\theta}\right)^{\beta}-1\right.}\right]\right]^{2}}=0
\end{aligned}
$$

where 


$$
\begin{aligned}
\pi_{5}= & \beta t_{i}^{\delta}\left[\ln \left(t_{i}\right)\right]\left(1+\frac{t_{i}^{\delta}}{\theta}\right)^{\beta-1} e^{-\lambda\left[\left(1+\frac{t_{i}^{\delta}}{\theta}\right)^{\beta}-1\right]} \\
& \left.\left\{1-e^{-\lambda\left[\left(1+\frac{t_{i}^{\delta}}{\theta}\right)^{\beta}-1\right]}\right\} 1-\lambda\left[\left(1+\frac{t_{i}^{\delta}}{\theta}\right)^{\beta}-1\right]\right\} \\
\pi_{6}= & \left.\lambda \beta t_{i}^{\delta}\left[\ln \left(t_{i}\right)\right]\left(1+\frac{t_{i}^{\delta}}{\theta}\right)^{\beta-1} e^{-2 \lambda\left[\left(1+\frac{t_{i}^{\delta}}{\theta}\right)^{\beta}-1\right]\left[\left(1+\frac{t_{i}^{\delta}}{\theta}\right)^{\beta}-1\right]}\right] \\
\frac{\partial \ell^{2}}{\partial \lambda \partial \theta}= & \left.\sum_{i=1}^{n} \frac{-\beta t_{i}^{\delta}}{\theta^{2}}\left(1+\frac{t_{i}^{\delta}}{\theta}\right)^{\beta-1}+(\gamma-1) \sum_{i=1}^{n} \frac{\pi_{7}+\pi_{8}}{\left[1-\lambda\left[\left(1+\frac{t_{i}^{\delta}}{\theta}\right)^{\beta}-1\right]\right.}\right]
\end{aligned}
$$

where

$$
\begin{aligned}
& \pi_{7}=\frac{\beta t_{i}^{\delta}}{\theta^{2}}\left(1+\frac{t_{i}^{\delta}}{\theta}\right)^{\beta-1} e^{-\lambda\left[\left(1+\frac{t_{i}^{\delta}}{\theta}\right)^{\beta}-1\right]}\left\{\lambda\left[\left(1+\frac{t_{i}^{\delta}}{\theta}\right)^{\beta}-1\right]-1\right\}\left\{1-e^{-\lambda\left[\left(1+\frac{t_{i}^{\delta}}{\theta}\right)^{\beta}-1\right]}\right\} \\
& \pi_{8}=\frac{-\beta \lambda t_{i}^{\delta}}{\theta^{2}}\left(1+\frac{t_{i}^{\delta}}{\theta}\right)^{\beta-1} e^{-2 \lambda\left[\left(1+\frac{t_{i}^{\delta}}{\theta}\right)^{\beta}-1\right]}\left[\left(1+\frac{t_{i}^{\delta}}{\theta}\right)^{\beta}-1\right] \\
& \frac{\partial \ell^{2}}{\partial \gamma^{2}}=\frac{-n}{\gamma^{2}}=0 \\
& \begin{array}{c}
\frac{\partial \ell^{2}}{\partial \gamma \partial \beta}=\sum_{i=1}^{n} \frac{\lambda\left(1+\frac{t_{i}^{\delta}}{\theta}\right)^{\beta} \ln \left(1+\frac{t_{i}^{\delta}}{\theta}\right) e^{-\lambda\left[\left(1+\frac{t_{i}^{\delta}}{\theta}\right)^{\beta}-1\right]}}{\left\{1-e^{\left.-\lambda\left[\left(1+\frac{t_{i}^{\delta}}{\theta}\right)^{\beta}-1\right]\right\}}\right.}=0 \\
\frac{\partial \ell^{2}}{\partial \gamma \partial \delta}=\sum_{i=1}^{n} \frac{\lambda \beta t_{i}^{\delta}\left(1+\frac{t_{i}^{\delta}}{\theta}\right)^{\beta-1} \ln \left(t_{i}\right) e^{-\lambda\left[\left(1+\frac{t_{i}^{\delta}}{\theta}\right)^{\beta}-1\right]}}{\left\{1-e^{\left.-\lambda\left[\left(1+\frac{t_{i}^{\delta}}{\theta}\right)^{\beta}-1\right]\right\}}\right.}=0
\end{array}
\end{aligned}
$$




$$
\begin{gathered}
\frac{\partial \ell^{2}}{\partial \gamma \partial \theta}=\sum_{i=1}^{n} \frac{\lambda \beta\left(1+\frac{t^{\delta}}{\theta}\right)^{\beta-1} e^{-\lambda\left[\left(1+\frac{t^{\delta}}{\theta}\right)^{\beta}-1\right]} t^{\delta}}{\left\{1-e^{-\lambda\left[\left(1+\frac{t_{i}^{\delta}}{\theta}\right)^{\beta}-1\right]}\right\} \theta^{2}}=0, \\
\frac{\partial \ell^{2}}{\partial \beta^{2}}=\frac{-n}{\beta^{2}}-2 \lambda \sum_{i=1}^{n}\left(1+\frac{t_{i}^{\delta}}{\theta}\right)^{\beta} \ln \left(1+\frac{t_{i}^{\delta}}{\theta}\right)+(\gamma-1) \sum_{i=1}^{n}\left[\frac{\rho_{1}+\rho_{2}}{\left\{e^{-\lambda\left[\left(1+\frac{t_{i}^{\delta}}{\theta}\right)^{\beta}-1\right]}\right]}\right]=0
\end{gathered}
$$

where

$$
\begin{aligned}
\rho_{1}= & \left\{1-e^{-\lambda\left[\left(1+\frac{t_{i}^{\delta}}{\theta}\right)^{\beta}-1\right]}\right\}\left\{\left(1+\frac{t_{i}^{\delta}}{\theta}\right)^{\beta} \ln \left(1+\frac{t_{i}^{\delta}}{\theta}\right) \lambda e^{-\lambda\left[\left(1+\frac{t_{i}^{\delta}}{\theta}\right)^{\beta}-1\right]}\right. \\
& \left.-\lambda\left(1+\frac{t_{i}^{\delta}}{\theta}\right)^{2 \beta} \ln \left(1+\frac{t_{i}^{\delta}}{\theta}\right) e^{-\lambda\left[\left(1+\frac{t_{i}^{\delta}}{\theta}\right)^{\beta}-1\right]}\right\} \\
\rho_{2}= & \left(1+\frac{t_{i}^{\delta}}{\theta}\right)^{2 \beta} \ln \left(1+\frac{t_{i}^{\delta}}{\theta}\right) \lambda e^{-2 \lambda\left[\left(1+\frac{t_{i}^{\delta}}{\theta}\right)^{\beta}-1\right]} \\
\frac{\partial \ell^{2}}{\partial \beta \partial \delta}= & \sum_{i=1}^{n} \frac{\ln (t) t_{i}^{\delta}}{\left(1+\frac{t^{\delta}}{\theta}\right)}-\lambda \sum_{i=1}^{n}\left\{\frac{t_{i}^{\delta}\left(1+\frac{t_{i}^{\delta}}{\theta}\right)^{\beta-1} \ln (t)}{\left(1+\frac{t^{\delta}}{\theta}\right)}\right. \\
& \left.+\ln \left(1+\frac{t_{i}^{\delta}}{\theta}\right) \beta t_{i}^{\delta} \ln (t)\left(1+\frac{t_{i}^{\delta}}{\theta}\right)^{\beta-1}\right\} \\
& +(\gamma-1) \sum_{i=1}^{n} \frac{\lambda\left(\pi_{9}\right)}{\pi_{10}^{2}}=0
\end{aligned}
$$

where 


$$
\begin{aligned}
& \pi_{9}=\left\{1-e^{-\lambda\left[\left(1+\frac{t_{i}^{\delta}}{\theta}\right)^{\beta}-1\right]}\right\}\left[1+\ln \left(1+\frac{t_{i}^{\delta}}{\theta}\right)^{\beta}+\lambda \ln \left(1+\frac{t_{i}^{\delta}}{\theta}\right)^{\beta}\right] \\
& \left\{\beta \ln \left(t_{i}\right) e^{-\lambda\left[\left(1+\frac{t_{i}^{\delta}}{\theta}\right)^{\beta}-1\right]} t_{i}^{\delta}\left(1+\frac{t_{i}^{\delta}}{\theta}\right)^{\beta}\right\} \\
& -\left(e^{-\lambda\left[\left(1+\frac{t_{i}^{\delta}}{\theta}\right)^{\beta}-1\right]}\right]\left[\left(1+\frac{t_{i}^{\delta}}{\theta}\right)^{\beta} \ln \left(1+\frac{t_{i}^{\delta}}{\theta}\right)^{\beta}+\beta \lambda t_{i}^{\delta} \ln \left(t_{i}\right)\left(1+\frac{t_{i}^{\delta}}{\theta}\right)^{\beta-1}\right] \\
& \pi_{10}=\left\{1-e^{-\lambda\left[\left(1+\frac{t_{i}^{\delta}}{\theta}\right)^{\beta}-1\right]}\right\} \text {. } \\
& \frac{\partial \ell^{2}}{\partial \beta \partial \theta}=-\sum_{i=1}^{n} \frac{t_{i}^{\delta}}{\theta^{2}\left(1+\frac{t_{i}^{\delta}}{\theta}\right)}+\lambda \sum_{i=1}^{n}\left\{\left(\left(1+\frac{t_{i}^{\delta}}{\theta}\right)^{\beta-1} \frac{t_{i}^{\delta}}{\theta^{2}}\right)\left[1+\beta \ln \left(1+\frac{t_{i}^{\delta}}{\theta}\right)\right]\right\} \\
& +(\gamma-1) \sum_{i=1}^{n} \frac{\lambda\left(\pi_{11}\right)}{\pi_{12}^{2}}=0
\end{aligned}
$$

where

$$
\begin{aligned}
& \pi_{11}=\left\{1-e^{-\lambda\left[\left(1+\frac{t_{i}^{\delta}}{\theta}\right)^{\beta}-1\right]}\right\}\{a+b+c\}-\lambda \beta t_{i}^{\delta}\left(1+\frac{t_{i}^{\delta}}{\theta}\right)^{2 \beta-1} e^{-2 \lambda\left[\left(1+\frac{t_{i}^{\delta}}{\theta}\right)^{\beta}-1\right]} \ln \left[t_{i}+1+\frac{t_{i}^{\delta}}{\theta}\right] \\
& a=\frac{-t_{i}^{\delta}\left(1+\frac{t_{i}^{\delta}}{\theta}\right)^{\beta-1} e^{-\lambda\left[\left(1+\frac{t_{i}^{\delta}}{\theta}\right)^{\beta}-1\right]}}{\theta^{2}}, \\
& b=\frac{-\beta t_{i}^{\delta}\left(1+\frac{t_{i}^{\delta}}{\theta}\right)^{\beta-1} e^{-\lambda\left[\left(1+\frac{l_{i}^{\delta}}{\theta}\right)^{\beta}-1\right]} \ln \left(1+\frac{t_{i}^{\delta}}{\theta}\right)}{\theta^{2}}, \\
& c=\frac{\lambda \beta t_{i}^{\delta}\left(1+\frac{t_{i}^{\delta}}{\theta}\right)^{2 \beta-1} e^{-\lambda\left[\left(1+\frac{t_{i}^{\delta}}{\theta}\right)^{\beta}-1\right]} \ln \left(1+\frac{t_{i}^{\delta}}{\theta}\right)}{\theta^{2}}, \\
& \pi_{12}=\left\{1-e^{-\lambda\left[\left(1+\frac{t_{i}^{\delta}}{\theta}\right)^{\beta}-1\right]}\right\} \text {. }
\end{aligned}
$$


$\frac{\partial \ell^{2}}{\partial \delta^{2}}=-\frac{n}{\delta^{2}}+(\beta-1) \sum_{i=1}^{n} 2 t^{\delta} \ln \left(t_{i}\right)\left[1+t_{i}^{\delta}\right]-\lambda \sum_{i=1}^{n}\{\tau\}+(\gamma-1) \sum_{i=1}^{n} \frac{\pi_{13}}{\pi_{14}^{2}}=0$

where

$$
\begin{aligned}
& \tau=\left[1+\beta \ln \left(1+\frac{t_{i}^{\delta}}{\theta}\right)\right] \\
& {\left[t_{i}^{\delta}\left(1+\frac{t_{i}^{\delta}}{\theta}\right)^{\beta-1} \ln \left(t_{i}\right)\right]} \\
& \pi_{13}=\left\{1-e^{-\lambda\left[\left(1+\frac{t_{i}^{\delta}}{\theta}\right)^{\beta}-1\right]}\right\}\{d+e+f\} \\
& -\left\{\ln (t) t_{i}^{\delta}\left(1+\frac{t_{i}^{\delta}}{\theta}\right)^{\beta-1} \beta \lambda e^{-\lambda\left[\left(1+\frac{t_{i}^{\delta}}{\theta}\right)^{\beta}-1\right]}\right\}^{2} \\
& d=-\ln \left(t_{i}\right)\left(t_{i}\right)^{2 \delta}\left(1+\frac{t_{i}^{\delta}}{\theta}\right)^{2 \beta-2} \beta \lambda e^{-\lambda\left[\left(1+\frac{t_{i}^{\delta}}{\theta}\right)^{\beta}-1\right]}, \\
& e=\ln \left(t_{i}\right)\left(t_{i}\right)^{2 \delta}\left(1+\frac{t_{i}^{\delta}}{\theta}\right)^{\beta-2}(\beta-1) e^{-\lambda\left[\left(1+\frac{t_{i}^{\delta}}{\theta}\right)^{\beta}-1\right]} \text {, } \\
& c=\ln \left(t_{i}\right)\left(t_{i}\right)^{\delta}\left(1+\frac{t_{i}^{\delta}}{\theta}\right)^{\beta-1} e^{-\lambda\left[\left(1+\frac{t_{i}^{\delta}}{\theta}\right)^{\beta}-1\right]} \text {, } \\
& \pi_{14}=\left\{1-e^{-\lambda\left[\left(1+\frac{t_{i}^{\delta}}{\theta}\right)^{\beta}-1\right]}\right\} \text {. } \\
& \frac{\partial \ell^{2}}{\partial \theta^{2}}=\frac{n}{\theta^{2}}+(\beta-1) \sum_{i=1}^{n} \frac{2 t_{i}^{\delta}}{\theta^{3}}-\lambda \sum_{i=1}^{n} \frac{2 t_{i}^{\delta}}{\theta^{3}}+(\gamma-1) \sum_{i=1}^{n} \frac{\pi_{15}}{\pi_{16}^{2}}=0
\end{aligned}
$$

where 


$$
\begin{aligned}
& \pi_{15}=\left\{1-e^{-\lambda\left[\left(1+\frac{t_{i}^{\delta}}{\theta}\right)^{\beta}-1\right]}\right\}\{g+h+i\} \\
& -\left\{\frac{\left(1+\frac{t_{i}^{\delta}}{\theta}\right)^{\beta-1} e^{-\lambda\left[\left(1+\frac{t_{i}^{\delta}}{\theta}\right)^{\beta}-1\right]}}{\theta^{2}}\right\} \\
& \left\{-\frac{\beta \lambda t_{i}^{\delta}\left(1+\frac{t_{i}^{\delta}}{\theta}\right)^{\beta-1} e^{-\lambda\left[\left(1+\frac{t_{i}^{\delta}}{\theta}\right)^{\beta}-1\right]}}{\theta^{2}}\right\} \\
& g=\frac{\beta \lambda t_{i}^{\delta}\left(1+\frac{t_{i}^{\delta}}{\theta}\right)^{2 \beta-2} e^{-\lambda\left[\left(1+\frac{t_{i}^{\delta}}{\theta}\right)^{\beta}-1\right]}}{\theta^{4}}, \\
& h=\frac{-(\beta-1) \lambda t_{i}^{\delta}\left(1+\frac{t_{i}^{\delta}}{\theta}\right)^{\beta-2} e^{-\lambda\left[\left(1+\frac{t_{i}^{\delta}}{\theta}\right)^{\beta}-1\right]}}{\theta^{4}} \\
& i=-\frac{2\left(1+\frac{t_{i}^{\delta}}{\theta}\right)^{\beta-1} e^{-\lambda\left[\left(1+\frac{t_{i}^{\delta}}{\theta}\right)^{\beta}-1\right]}}{\theta^{3}}, \\
& \pi_{16}=\left\{1-e^{-\lambda\left[\left(1+\frac{t_{i}^{\delta}}{\theta}\right)^{\beta}-1\right]}\right\} \\
& \frac{\partial \ell^{2}}{\partial \theta \partial \delta}=-(\beta-1) \sum_{i=1}^{n} \frac{t_{i}^{\delta} \ln (t)}{\theta^{2}}+\lambda \sum_{i=1}^{n} \frac{t_{i}^{\delta} \ln \left(t_{i}\right)}{\theta^{2}}+\beta \lambda(\gamma-1) \sum_{i=1}^{n} \frac{\pi_{17}}{\pi_{18}^{2}}=0
\end{aligned}
$$

where 


$$
\begin{aligned}
\pi_{17} & =\theta^{2}\left\{1-e^{-\lambda\left[\left(1+\frac{t_{i}^{\delta}}{\theta}\right)^{\beta}-1\right]}\right\}\{j+k+l\}-\left\{t_{i}^{\delta}\left(1+\frac{t_{i}^{\delta}}{\theta}\right)^{\beta-1} e^{-\lambda\left[\left(1+\frac{t_{i}^{\delta}}{\theta}\right)^{\beta}-1\right]} \rho_{3}\right. \\
j & =\ln \left(t_{i}\right)\left(t_{i}\right)^{2 \delta}\left(1+\frac{t_{i}^{\delta}}{\theta}\right)^{2 \beta-2}(\beta-1) e^{-\lambda\left[\left(1+\frac{t_{i}^{\delta}}{\theta}\right)^{\beta}-1\right],} \\
k & =\ln \left(t_{i}\right)\left(t_{i}\right)^{2 \delta}\left(1+\frac{t_{i}^{\delta}}{\theta}\right)^{\beta-2}(\beta-1) e^{-\lambda\left[\left(1+\frac{t_{i}^{\delta}}{\theta}\right)^{\beta}-1\right],} \\
l & =\ln \left(t_{i}\right) t_{i}^{\delta}\left(1+\frac{t_{i}^{\delta}}{\theta}\right)^{\beta-1} e^{-\lambda\left[\left(1+\frac{t_{i}^{\delta}}{\theta}\right)^{\beta}-1\right]}, \\
\pi_{18} & =\theta^{2}\left\{1-e^{-\lambda\left[\left(1+\frac{t_{i}^{\delta}}{\theta}\right)^{\beta}-1\right]}\right\} \cdot \rho_{3}=\left\{\theta^{2} \ln (t) t_{i}^{\delta}\left(1+\frac{t_{i}^{\delta}}{\theta}\right)^{\beta-1}(\beta-1) e^{-\lambda\left[\left(1+\frac{t_{i}^{\delta}}{\theta}\right)^{\beta}-1\right]}\right\}
\end{aligned}
$$

Funding No funding was involved in the present work. The authors did not receive support from any organization for the submitted work. The authors have no financial or proprietary interests in any material discussed in this article.

Data and code availability statement All the data and codes used in this study, as well as, the supplementary material can be made available from the corresponding author, upon reasonable request.

\section{Compliance with Ethical Standards}

Involvement of human participant and animals this article does not contain any studies with animals or humans performed by any of the authors. Information about informed consent No informed consent was required as the studies does not involve any human participant.

Author contributions Conceptualization, experiments/code executions, the formal analysis and Review \& editing was done by Hanem Mohamed, Salwa Mousa, Amina Esmail Abo-Hussien and Magda Mohamed Ismail.

Ethic statements All authors consciously assure that for the manuscript fulflls the following statements: 1) This material is the authors' own original work, which has not been previously published elsewhere. 2) The paper is not currently being considered for publication elsewhere. 3) The paper refects the authors' own research and analysis in a truthful and complete manner. 4) The paper properly credits the meaningful contributions of co-authors and co-researchers. 5) The results are appropriately placed in the context of prior and existing research.

Conflict of interest The authors declare no conflict of interest.

\section{References}

1. Kumar S (2020) Monitoring novel corona virus (COVID-19) infections in india by cluster analysis. Ann Data Sci 7:417-425 
2. Khakharia A, Shah V, Jain S, Shah J, Tiwari A, Daphal P, Mehendale N (2021) Outbreak prediction of COVID-19 for dense and populated countries using machine learning. Ann Data Sci 8(1):1-19

3. Olson DL, Shi Y (2007) Introduction to business data mining. McGraw-Hill/Irwin, New York

4. Shi Y, Tian YJ, Kou G, Peng Y, Li JP (2011) Optimization based data mining: theory and applications. Springer, Berlin

5. Li J, Guo K, Herrera Viedma E, Lee H, Liu J, Zhong Z, Gomes L, Filip FG, Fang SC, Özdemir MS, Liu XH, Lu G, Sh Y (2020) Culture vs policy: more global collaboration to effectively combat COVID-19. The Innovation. https://doi.org/10.1016/j.xinn.2020.100023

6. Liu Y, Gu Z, Xia S, Shi B, Zhou X, Shi Y, Liu J (2020) What are the underlying transmission patterns of COVID-19 outbreak an age-specific social contact characterization. E Clin Med 22:100354

7. Datta R, Trivedi PK, Kumawat A, Kumar R, Bhardwaj I, Kumari N, Agiwal V, Kumar S, Kumar A, Shukla A, Kumar J (2020) Statistical modeling of COVID-19 pandemic stages worldwide. Preprints 2020, 2020050319. https://doi.org/10.20944/preprints202005.0319.v1

8. Villalobos-Arias M (2020) Using generalized logistics regression to forecast population infected by Covid-19, arXiv preprint arXiv:2004.02406

9. El-Bassiouny AH, Abdo NF, Shahen HS (2015) Exponential Lomax distribution. Int J Comput Appl 121(13):24-29

10. Hassan AS, Abd-Alla M (2018) Exponentiated Weibull Lomax: properties and estimation. J Data Sci 16:277-298. https://doi.org/10.6339/jds.201804_16(2).0004

11. Cordeiro GM, Afify AZ, Ortega EM, Suzuki AK, Mead ME (2019) The odd Lomax generator of distributions: properties, estimation and applications. J Comput Appl Math 347:222-237. https:// doi.org/10.1016/j.cam.2018.08.008

12. Chesneau C, Djlbrila S (2019) The generalized odd inverted exponential-G family of distributions: properties and applications. Eurasian Bull Math 2(3):86-110

13. Alizadeh M, Afify AZ, Eliwa M, Ali S (2020) The odd log-logistic Lindley-G family of distributions: properties, Bayesian and non-Bayesian estimation with applications. Comput Stat 35:281-308

14. Afify A, Alizadeh M (2020) The odd dagum family of distributions: properties and applications. J Appl Prob Stat 15:45-72

15. Gupta RD, Kundu D (1999) Generalized exponential distributions. Aust N Z J Stat 41(2):173-188

16. Gupta RD, Kundu D (1999b) Generalized exponential distributions: Statistical Inferences, Technical Report, the University of New Brunswick, Saint John

17. Lomax KS (1954) Business failures; another example of the analysis of failure data. J Am Stat Assoc 49:847-852

18. Alzaatreh A, Lee C, Famoye F (2013) A new method for generating families of distributions. Metron 71:63-79

19. Hanem M, Amina EA-H, Salwa AMM, Ismail M (2021) The Odd generalized exponential Lomax distribution properties and estimation. J Mod Appl Stat Methods (In press)

20. Ghitany M, Al-Mutairi D, Balakrishnan N, Al-Enezi I (2013) Power Lindley distribution and associated inference. Comput Stat Data Anal 64:20-33

21. Assar SM (2018) On odds generalized exponential-power Lomax distribution. J Math Stat 14(1):167-174. https://doi.org/10.3844/jmssp.2018.167.174

22. Casella G, Berger RL (1990) Statistical inference. Brooks/Cole Publishing Company, Pacific Grove

23. Thongkairat S, Yamaka W, Sriboonchitta S (2018) Maximum product spacings method for the estimation of parameters of linear regression. J Phys: Conf Ser 1053:012110

24. Cheng RCH, Amin NAK (1983) Estimating parameters in continuous univariate distributions with a shifted origin. J R Stat Soc Ser B Methodol 45(3):394-403

25. Anatolyev S, Kosenok G (2005) An alternative to maximum likelihood based on spacings. Economet Theor 21(2):472-476

26. Aarset MV (1987) How to identify a bathtub hazard rate. IEEE Trans Reliab R-36(1):106-108. https://doi.org/10.1109/TR.1987.5222310

Publisher's Note Springer Nature remains neutral with regard to jurisdictional claims in published maps and institutional affiliations. 